\title{
Wang-Landau sampling for quantum systems: algorithms to overcome tunneling problems and calculate the free energy
}

\author{
Matthias Troyer ${ }^{(1,2)}$, Stefan Wessel ${ }^{(1)}$, and Fabien Alet ${ }^{(3,1,2)}$ \\ (1) Theoretische Physik, ETH Zürich, CH-8093 Zürich, Switzerland \\ ${ }^{(2)}$ Computation Laboratory, ETH Zürich, CH-8092 Zürich, Switzerland and \\ ${ }^{(3)}$ Laboratoire de Physique Quantique, Université Paul Sabatier, 31062 Toulouse, France
}

(Dated: July 19, 2021)

\begin{abstract}
We present a generalization of the classical Wang-Landau algorithm [Phys. Rev. Lett. 86, 2050 (2001)] to quantum systems. The algorithm proceeds by stochastically evaluating the coefficients of a high temperature series expansion or a finite temperature perturbation expansion to arbitrary order. Similar to their classical counterpart, the algorithms are efficient at thermal and quantum phase transitions, greatly reducing the tunneling problem at first order phase transitions, and allow the direct calculation of the free energy and entropy.
\end{abstract}

PACS numbers: 02.70.Ss,05.10.Ln,05.30.-d,75.40.Mg

Monte Carlo simulations in statistical physics now have a history of nearly half a century starting with the seminal work of Metropolis [1]. While the Metropolis algorithm has been established as the standard algorithm for importance sampling it suffers from two problems: the inability to directly calculate the partition function, free energy or entropy, and critical slowing down near phase transitions and in disordered systems.

In a standard Monte Carlo algorithm a series of configurations is generated according to a given distribution, usually the Boltzmann distribution in classical simulations. While this allows the calculation of thermal averages, it does not give the partition function, nor the free energy. They can only be obtained with limited accuracy as a temperature integral of the specific heat, or by using maximum entropy methods [2].

The problem of critical slowing down has been overcome for second order phase transitions by cluster update schemes for classical [3] and quantum systems [4, 5, 6, 7. For first order phase transitions and systems with rough free energy landscapes a decisive improvement was achieved recently by a new algorithm for classical systems due to Wang and Landau [10]. In contrast to related methods - such as the multicanonical [8] or the broad histogram [9] method - this new algorithm scales to large systems, does not suffer from systematic errors and needs no a priori knowledge. The key idea is to calculate the density of states $\rho(E)$ directly by a random walk in energy space instead of performing a canonical simulation at a fixed temperature. By visiting each energy level $E$ with a probability $1 / \rho(E)$, this algorithm achieves a flat histogram and good precision over the whole energy range. Besides being efficient at first and second order phase transitions this algorithm allows the direct calculation of the free energy from the partition function $Z=\sum_{E} \rho(E) e^{-E / k_{B} T}$. The internal energy, entropy, specific heat and other thermal properties are easily obtained as well, by differentiating the free energy. Within a year of publication this algorithm has been improved using $N$-fold way [11 and multibondic 112 sampling schemes, has been applied to Potts models 113 and generalized to reaction coordinates 14], continuum models [15], polymer films 16], and to protein folding [17].

Since simulations of quantum systems suffer from the same problems as classical simulations, in particular from long tunneling times at first order phase transitions and the inability to calculate the free energy directly, an extension of this algorithm to quantum systems is highly desired. Here we present two such algorithms. The first one is based upon a high temperature series expansion. Similarly to the classical algorithm it allows the calculation of the free energy as a function of temperature, making it ideal for the study of thermal phase transitions. The second algorithm renders the high temperature series expansion into a perturbation expansion in one of the coupling constants and is suitable for the investigation of quantum phase transitions.

Quantum Monte Carlo algorithms start by mapping the quantum system to a classical system. This can be done either through a discrete [18] or continuous time [5] path integral representation or by a stochastic series expansion (SSE) in the inverse temperature [19]. While our algorithms can be formulated in both representations, we here present the SSE version as it is the easiest and most natural representation for most problems.

We start by expressing the partition function as a high temperature expansion

$$
Z=\operatorname{Tr} e^{-\beta H}=\sum_{n=0}^{\infty} \frac{\beta^{n}}{n !} \operatorname{Tr}(-H)^{n} \equiv \sum_{n=0}^{\infty} g(n) \beta^{n},
$$

where the $n$-th order series coefficient $g(n)=$ $\operatorname{Tr}(-H)^{n} / n$ ! will play the role of the density of states in the classical algorithm. The algorithm performs a random walk in the space of series expansion coefficients, achieves a flat histogram in their orders $n$ and calculates the coefficients $g(n)$. Employing Eq. (1) we can then calculate the free energy, internal energy, entropy and spe- 
cific heat directly. Thermal averages of observables can be measured as in conventional Monte Carlo algorithms by recording a separate histogram for the expectation values at each order.

Next we note that in a computer simulation the series expansion (11) needs to be truncated at an order $\Lambda$. Since the orders relevant for a given inverse temperature $\beta$ are sharply peaked around $\beta|E(\beta)|$, where $E(\beta)$ is the mean energy at inverse temperature $\beta$, this cutoff does not introduce a systematic error. Its main consequence is to restrict the accessible temperature range to $\beta \lesssim \Lambda / E(\beta)$.

The next step is to introduce a complete set of basis states $\{|\alpha\rangle\}$, and to decompose the Hamiltonian $H$ into diagonal and offdiagonal bond terms $H_{b}^{(a)}$. For simplicity we restrict the following discussion to a spin- $1 / 2$ Heisenberg model where this decomposition for a bond $b=(i, j)$ reads $H_{(i, j)}^{(d)}=J S_{i}^{z} S_{j}^{z}-J / 4$, and $H_{(i, j)}^{(o)}=$ $J / 2\left(S_{i}^{+} S_{j}^{-}+S_{i}^{-} S_{j}^{+}\right)$. The offset $-J / 4$ is added to the diagonal part in order to render the matrix elements of $-H$ nonnegative. Using this decomposition, we can write the partition function as [6, 19]

$$
Z=\sum_{\alpha} \sum_{\left\{S_{\Lambda}\right\}} \frac{\beta^{n}(\Lambda-n) !}{\Lambda !}\left\langle\alpha\left|\prod_{i=0}^{\Lambda}\left(-H_{b_{i}}^{\left(a_{i}\right)}\right)\right| \alpha\right\rangle,
$$

where the operator string $S_{\Lambda}=\left(\left(b_{1}, a_{1}\right), \ldots,\left(b_{\Lambda}, a_{\Lambda}\right)\right)$ is a concatenation of $n$ bond operators and $\Lambda-n$ unit operators.

Comparing Eq. (11) to Eq. (2) we see that we can obtain $g(n)$ by counting the number of times a configuration with $n$ non-unit operators is observed during a simulation at an inverse temperature $\beta=1$. As the dynamic range of $g(n)$ spans thousands of orders of magnitude $\left[g(0) / g(\Lambda)\right.$ is up to $10^{10000}$ for the examples given below] simply collecting a histogram will not be effective. Therefore a variant of the classical Wang-Landau method [10] will be employed: by reweighting a configuration of $n$-th order with $1 / g(n)$ a flat histogram of the orders $n$ can be achieved, thus sampling all orders equally well.

Since $g(n)$ is initially unknown we start with the (bad) guess $g(n)=1$. Each time a configuration of $n$-th order is visited, $g(n)$ is multiplied by a factor $f$, i.e. $g(n) \leftarrow$ $f g(n)$. In our implementation we store the logarithms of these quantities to avoid overflow problems. The random walk is performed until the histogram $H(n)$ - counting the number of times a configuration with $n$ operators is observed - is reasonably flat. Similar to the classical case a maximum deviation of $20 \%$ from the mean value turned out to be reasonable. The multiplicative increase of $g(n)$ is essential for the success of the algorithm. Only that way the large range of $g(n)$ can be mapped out in reasonable time, and $g(n)$ converges rapidly to a rough estimate of the true distribution. Once the histogram is flat, it is reset to zero, $f$ is decreased, in our case by $f \leftarrow \sqrt{f}$, and the process starts again, refining $g(n)$

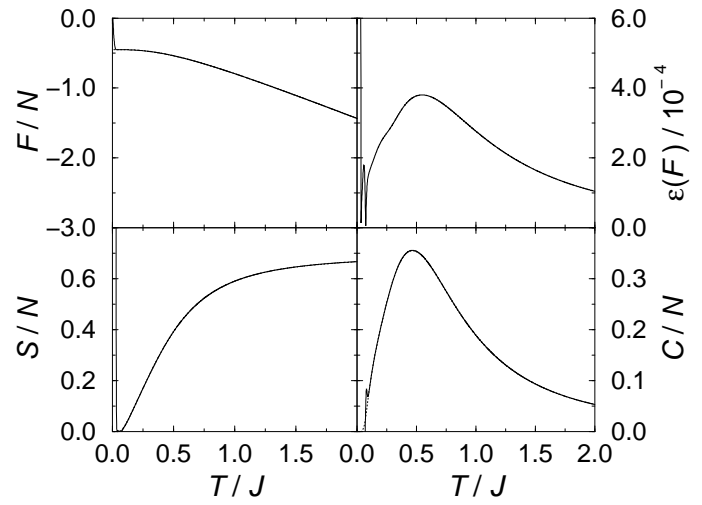

FIG. 1: Free energy $F$, entropy $S$ and specific heat $C$ of an $N=10$ site antiferromagnetic Heisenberg chain. Solid lines correspond to the MC results, indistinguishable from the dotted lines for the exact results. Also shown is the relative error $\varepsilon(F)$ of $F$ compared to the exact result.

further with smaller steps. This procedure is repeated until $f$ gets as small as $\exp \left(10^{-8}\right)$, so that an accurate estimate of $g(n)$ with only negligible systematic errors will be available. The accuracy of the free energy and other calculated quantities is given by the statistical error which, as usual, scales with $1 / \sqrt{N_{\mathrm{MC}}}$ where $N_{\mathrm{MC}}$ is the number of Monte Carlo steps. The overall normalization of $g(n)$ follows from $g(0)$ being equal to the dimension of the Hilbert space, which for a spin-1/2 Heisenberg model on a lattice with $N$ sites is $2^{N}$. The initial choice of $f$ is very important. Too small starting values result in long computation times, while too large values give extreme fluctuations in the initial iterations. As in the classical Wang-Landau method a good choice is to let $f^{N_{\mathrm{MC}}}$ be of the same order of magnitude as the total number of configurations, which is of order $2^{N} N^{\Lambda}$. Since usually $\Lambda \gg N$, a good initial value is $\ln f \approx(\Lambda \ln N) / N_{\mathrm{MC}}$.

To finish the description of the algorithm we discuss the update steps in more detail. Any of the known update algorithms, employing local 19], or cluster [6] updates can be used. The only change in the acceptance probabilities from standard SSE algorithms is to set $\beta=1$ and to include an additional factor $g(n) / g\left(n^{\prime}\right)$ in the acceptance probability for any move that changes the number of operators from $n$ to $n^{\prime}$. As an example we discuss the Heisenberg antiferromagnet. There the optimal algorithm is the loop algorithm, which in the SSE representation consists of two parts: diagonal updates and loop updates. In a diagonal update step the operator string positions $l=1, \ldots, \Lambda$ are traversed in ascending order. Empty and diagonal operators can be exchanged with each another. Using the notation $|\alpha(l)\rangle=\prod_{i=1}^{l} H_{b_{i}}^{\left(a_{i}\right)}|\alpha\rangle$ for the state obtained by acting on $|\alpha\rangle$ with the first $l$ bond operators, and $M$ for the total number of interact- 


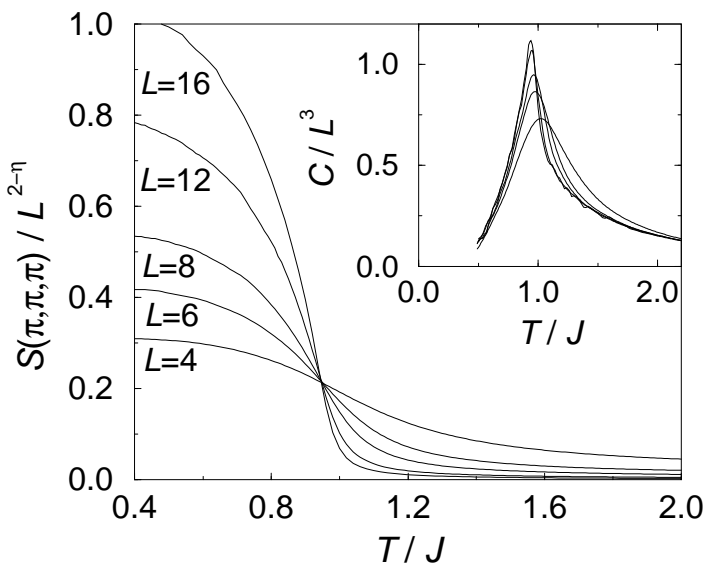

FIG. 2: Scaling plot of the staggered structure factor of a cubic antiferromagnet as a function of temperature, obtained from simulations at a fixed temperature for various lattice sizes. The inset shows the specific heat as a function of temperature. The cutoff $\Lambda=500(L / 4)^{3}$ restricts the accessible temperature range to $T \gtrsim 0.4 J$.

ing bonds on the lattice, the update probabilities are

$$
\begin{aligned}
& P\left[H^{(0)}(l) \rightarrow H_{b}^{(d)}(l)\right]=\frac{g(n) M\left\langle\alpha(l)\left|H_{b}^{(d)}\right| \alpha(l)\right\rangle}{g(n+1)(\Lambda-n)}, \\
& P\left[H_{b}^{(d)}(l) \rightarrow H^{(0)}(l)\right]=\frac{g(n)(\Lambda-n+1)}{g(n-1) M\left\langle\alpha(l)\left|H_{b}^{(d)}\right| \alpha(l)\right\rangle},
\end{aligned}
$$

where $P>1$ is interpreted as $P=1$. This choice of update probabilities requires the least changes to an existing SSE program. Alternatively the factors $M, \Lambda-n$ and $\Lambda-n+1$ can be dropped from the update probabilities, thus simplifying the algorithm. To correct for this omission, the obtained $g(n)$ must then be multiplied by $M^{n}(\Lambda-n) ! / \Lambda$ !. At each level $l$, independent of whether an update was performed or not (e.g. when the operator is off-diagonal) $g(n)$ for the current value of $n$ is incremented by $f$. The second part of the update cycle, the loop update, changes diagonal to off-diagonal bond operators without changing $n$ and can be performed as in standard SSE algorithms. We refer to Ref. [6] for details.

As a first example we show in Fig. 11 results of calculations for the free energy $F$, entropy $S$ and specific heat $C$ of an $N=10$ site antiferromagnetic Heisenberg chain, and compare to exact results. Using $10^{8}$ sweeps, which can be performed in less than five hours on an $800 \mathrm{MHz}$ Pentium-III CPU, the errors can be reduced down to the order of $10^{-4}$. The cutoff was set to $\Lambda=250$, restricting the accessible temperatures to $T \gtrsim 0.05 \mathrm{~J}$. The sudden departure of the Monte Carlo data from the exact values below this temperature clearly shows this limit, which can be pushed lower by increasing $\Lambda$. The sudden deviation becomes even more pronounced in larger systems and provides a reliable indication for the range of validity of the results.

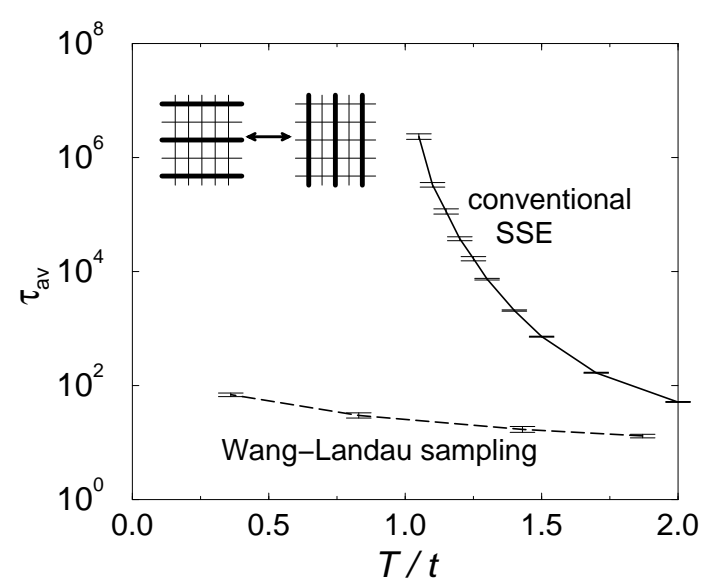

FIG. 3: Average tunneling times (in units of Monte Carlo sweeps) between horizontal and vertical arrangement of stripes in a hard core boson model at a ratio $V_{2} / t=3$ of next nearest neighbor repulsion to kinetic energy on a $8 \times 8$ sites lattice. The solid line is obtained using the standard SSE algorithm with directed loop updates. The dashed line is obtained using our algorithm, where the temperature is defined as the lowest temperature accessible in the simulation.

To illustrate the efficiency of the algorithm close to a thermal second order phase transition, we consider the Heisenberg antiferromagnet on a simple cubic lattice. From simulations of systems with $L^{3}$ sites, $L=$ $4,6,8,12,16$, we can calculate the staggered structure factor $S(\pi, \pi, \pi)$ for any value of the temperature using the measured histograms. Figure 2 shows the scaling plot of $S(\pi, \pi, \pi) / L^{2-\eta}$ with $\eta=0.034$. The estimate for the critical temperature $T_{c}=0.947 \mathrm{~J}$, obtained in only a couple of days on an $800 \mathrm{MHz}$ Pentium-III CPU, compares well with earlier estimates [20].

Next we demonstrate the efficiency of the algorithm at a first order phase transition by considering twodimensional hardcore bosons with next-nearest neighbour interactions [21]. At low temperature and half filling this model is in an insulating phase with striped charge order and provides the simplest quantum mechanical model with stripes. We are currently investigating the thermal and quantum melting transitions of this stripe phase and probe for the existence of a nematic phase [22]. Simulations with conventional update schemes suffer from exponentially increasing tunneling times needed to change the stripe orientation from a horizontal to a vertical arrangement. The flat histogram in the order $n$ in our algorithm reduces the tunneling times by many orders of magnitude already on small lattices (c.f. Fig. 3) which demonstrates the efficiency of our algorithm at first order phase transitions.

We now turn to our second algorithm, which applies to quantum phase transitions. Instead of scanning a temperature range we vary one of the interactions at fixed temperature. Defining the Hamiltonian as $H=H_{0}+\lambda V$ 


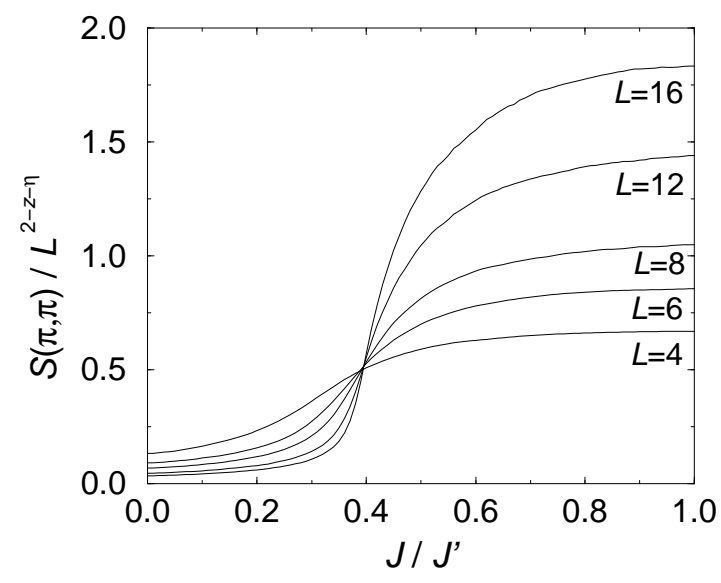

FIG. 4: Scaling plot of the staggered structure factor of a Heisenberg bilayer as a function of the coupling ratio $\lambda=$ $J / J^{\prime}$. Results are shown for various linear system sizes $L$. The temperature was chosen $\beta J^{\prime}=2 L$, low enough to be in the scaling regime. The cutoff $\Lambda=8 L^{3}$ was chosen large enough to cover the coupling range $J / J^{\prime} \lesssim 1$. The dynamical critical exponent of this model is $z=1$ and $\eta=0.034$.

we rewrite the partition function Eq. (1) as

$$
Z=\sum_{n=0}^{\infty} \frac{\beta^{n}}{n !} \operatorname{Tr}\left(-H_{0}-\lambda V\right)^{n} \equiv \sum_{n_{\lambda}=0}^{\infty} \tilde{g}\left(n_{\lambda}\right) \lambda^{n_{\lambda}},
$$

where on the right hand side we have collected all terms associated with $\lambda^{n_{\lambda}}$ into $\tilde{g}\left(n_{\lambda}\right)$. A similar algorithm can now be devised for this perturbation expansion up to arbitrary orders by setting $\lambda=1$, replacing $M$ by $\beta M$ and $g(n)$ by $\tilde{g}\left(n_{\lambda}\right)$ in Eq. (3). To normalize $\tilde{g}\left(n_{\lambda}\right)$ there are two options. If $H_{0}$ can be solved exactly, $\tilde{g}(0)$ can be determined directly. Otherwise, the normalization can be fixed using the first algorithm to calculate $Z(\beta)$ at any fixed value of $\lambda$. Finally, even without normalization we can still obtain entropy and energy differences.

We consider as an example the quantum phase transition in a bilayer Heisenberg antiferromagnet whose ground state changes from quantum disordered to Néel ordered as the ratio $\lambda=J / J^{\prime}$ of intra-plane $(J)$ to inter-plane $\left(J^{\prime}\right)$ coupling is increased [23]. From the histograms generated within one simulation we can calculate the staggered structure factor $S(\pi, \pi)$ of the system at any value of $\lambda$. In Fig. 4 we show a scaling plot of $S(\pi, \pi) / L^{2-z-\eta}$ as a function of $\lambda$. In short simulations, taking only a few days on an $800 \mathrm{MHz}$ Pentium-III CPU, we find the quantum critical point at $\lambda=0.396$, which again compares well with earlier results [23].

To summarize, we have presented Monte Carlo algorithms for the direct calculation of the free energy of a quantum system, based on a stochastic series expansion representation of the partition function. Our algorithms employ a variant of the Wang-Landau sampling to achieve a flat histogram and provide the free energy as well as thermodynamic averages accurately over a whole temperature or coupling range. The algorithms can be used with any of the update schemes developed for the SSE representation and require only minor modifications to existing programs. Parallelization of the algorithms can be done like in the classical case by splitting the $n$ range into multiple random walks over a shorter range.

Our algorithms are efficient not only at second order phase transitions but also at first order ones, where standard local and cluster update methods fail. The algorithms open up new possibilities for quantum Monte Carlo simulations: similar to the classical case we expect the algorithms to be efficient also for disordered systems and work is in progress to apply the methods to quantum spin glasses. Also, the ability to calculate the free energy and entropy will be useful for investigations of entropydriven phase transitions, such as the reentrant melting transition observed in bosonic systems and anisotropic quantum magnets 24.

We thank D.P. Landau for discussions and acknowledge support of the Swiss National Science Foundation.

[1] N. Metropolis et al., J. Chem. Phys. 21, 1087 (1953).

[2] C. Huscroft et al., Phys. Rev. B 61, 9300 (2000).

[3] R.H. Swendsen and J.-S. Wang, Phys. Rev. Lett. 58, 86 (1987); U. Wolff, Phys. Rev. Lett. 62, 361 (1989).

[4] H.G. Evertz et al., Phys. Rev. Lett. 70, 875 (1993).

[5] B. B. Beard and U. J. Wiese, Phys. Rev. Lett. 77, 5130 (1996).

[6] A.W. Sandvik, Phys. Rev. B 59, R14157 (1999); O. F. Syljuåsen and A. W. Sandvik, Phys. Rev. E 66, 046701 (2002).

[7] N. V. Prokof'ev et al., Sov. Phys. - JETP 87, 310 (1998).

[8] B.A. Berg and T. Neuhaus, Phys. Rev. Lett. 68, 9 (1992).

[9] P.M.C. de Oliveira et al., J. Phys. 26, 677 (1996).

[10] F. Wang and D.P. Landau, Phys. Rev. Lett. 86, 2050 (2001); Phys. Rev. E 64, 056101 (2001).

[11] B.J. Schulz et al., Int. J. Mod. Phys. C 13, 477 (2002).

[12] C. Yamaguchi and Naoki Kawashima, Phys. Rev. E 65, 056710 (2002).

[13] C. Yamaguchi and Y. Okabe, J. Phys. A 34, 8781 (2001).

[14] F. Calvo, Molec. Phys 100, 3421 (2002).

[15] Q. Yan et al., J. Chem. Phys. 116, 8745 (2002); M.S. Shell et al., Report cond-mat/0206461.

[16] T.S. Jain et al., J. Chem. Phys. 116, 7238 (2002).

[17] N. Rathore et al., J. Chem. Phys. 116, 7225 (2002).

[18] M. Suzuki, Prog. Theor. Phys. 65, 1454 (1976).

[19] A.W. Sandvik and J. Kurkijärvi, Phys. Rev. B 43, 5950 (1991); A. W. Sandvik, J. Phys. A 25, 3667 (1992).

[20] A.W. Sandvik, Phys. Rev. Lett. 80, 5196 (1998).

[21] F. Hebert et al., Phys. Rev. B 65, 014513 (2002).

[22] G. Schmid et al. in Computer Simulation Studies in Condensed Matter Physics XV, ed. D.P. Landau et al. (Springer Verlag Berlin Heidelberg,2002).

[23] A. W. Sandvik and D.J. Scalapino, Phys. Rev. Lett. 72, 2777 (1994).

[24] G. Schmid et al., Phys. Rev. Lett. 88, 167208 (2002). 